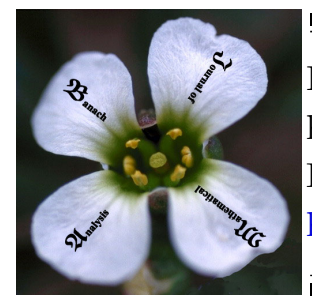

Banach J. Math. Anal. 9 (2015), no. 1, 111-126

http://doi.org/10.15352/bjma/09-1-9

ISSN: $1735-8787$ (electronic)

http://projecteuclid.org/bjma

\title{
REFINEMENTS OF QUASI-ARITHMETIC MEANS INEQUALITIES FOR HILBERT SPACE OPERATORS
}

\author{
JADRANKA MIĆIĆ
}

Communicated by M. Fujii

\begin{abstract}
In this paper some inequalities involving quasi-arithmetic means for a continuous field of self-adjoint operators, a field of positive linear mappings and continuous strictly monotone functions are refined. These refined converses are presented by using the Mond-Pečarić method improvement. Obtained results are applied to refine selected inequalities with power functions.
\end{abstract}

\section{INTRODUCTION}

Let $T$ be a locally compact Hausdorff space and $\mu$ be a Radon measure on $T$. Let $\mathcal{A}$ be a $C^{*}$-algebra of operators on some Hilbert space $H$. We say that a field $\left(x_{t}\right)_{t \in T}$ of operators in $\mathcal{A}$ is continuous if the function $t \mapsto x_{t}$ is norm continuous on $T$.

Assume further that there is a field $\left(\phi_{t}\right)_{t \in T}$ of positive linear mappings $\phi_{t}$ : $\mathcal{A} \rightarrow \mathcal{B}$ from $\mathcal{A}$ to another $\mathcal{C}^{*}$-algebra $\mathcal{B}$ of operators on a Hilbert space $K$. We say that such a field is continuous if the function $t \mapsto \phi_{t}(x)$ is continuous for every $x \in \mathcal{A}$. Additionally, if the $\mathcal{C}^{*}$-algebras include the identity operators and the function $t \mapsto \phi_{t}\left(1_{H}\right)$ is integrable with $\int_{T} \phi_{t}\left(1_{H}\right) d \mu(t)=1_{K}$, we say that a field $\left(\phi_{t}\right)_{t \in T}$ is unital.

Let $B(H)$ be the $C^{*}$-algebra of all bounded linear operators on a Hilbert space $H$. We define bounds of a self-adjoint operator $x \in B(H)$ by

$$
m_{x}:=\inf _{\|\xi\|=1}\langle x \xi, \xi\rangle \text { and } M_{x}:=\sup _{\|\xi\|=1}\langle x \xi, \xi\rangle
$$

Date: Received: Feb. 8, 2014; Accepted: Apr. 4, 2014.

2010 Mathematics Subject Classification. Primary 47A63; Secondary 47B15, 47A64.

Key words and phrases. Quasi-arithmetic mean, power mean, self-adjoint operator, positive linear mapping, Jensen's operator inequality. 
for $\xi \in H$.

The absolute value of $x \in B(H)$ is defined by $|x|:=\left(x^{*} x\right)^{1 / 2}$.

We observe the quasi-arithmetic operator mean

$$
\mathfrak{M}_{\varphi}(\mathbf{x}, \phi)=\varphi^{-1}\left(\int_{T} \phi_{t}\left(\varphi\left(x_{t}\right)\right) d \mu(t)\right)
$$

where $\left(x_{t}\right)_{t \in T}$ is a bounded continuous field of self-adjoint operators in a $C^{*}$ algebra $B(H)$ with spectra in $[m, M]$ for some scalars $m<M,\left(\phi_{t}\right)_{t \in T}$ is a unital field of positive linear mappings $\phi_{t}: B(H) \rightarrow B(K)$ and $\varphi \in \mathcal{C}[m, M]$ is a strictly monotone function. This mean is briefly denoted by $\mathfrak{M}_{\varphi}$.

In [6] the monotonicity of these means is presented:

$$
\mathfrak{M}_{\varphi} \leq \mathfrak{M}_{\psi}
$$

for all strictly monotone functions $\psi, \varphi \in \mathcal{C}[m, M]$, such that one of the following conditions holds:

(i): $\psi \circ \varphi^{-1}$ is operator convex and $\psi^{-1}$ is operator monotone

(ii): $\psi \circ \varphi^{-1}$ is operator concave and $-\psi^{-1}$ is operator monotone

(iii): $\varphi^{-1}$ is operator convex and $\psi^{-1}$ is operator concave

Monotonicity (1.2) without operator convexity is presented in [5], but with conditions on the bounds of $x_{t}, t \in T$. Improvements of some operator means inequalities are, for example, considered in [4].

The purpose of this paper is to consider converse of (1.2). We study refining of these inequalities without operator convexity or operator monotonicity and without conditions on spectra of the operators. Applying the obtained results we further refine selected inequalities involving power means.

\section{QUASI-ARITHMETIC MEANS}

For convenience we introduce some abbreviations. For $f: I \rightarrow \mathbb{R}$ and $m, M \in I$, $m \neq M$, we denote

$$
\delta_{f} \equiv \delta_{f}(m, M):=f(m)+f(M)-2 f\left(\frac{m+M}{2}\right)
$$

and

$$
k_{f} \equiv k_{f}(m, M):=\frac{f(M)-f(m)}{M-m} \quad \text { and } \quad l_{f} \equiv l_{f}(m, M):=\frac{M f(m)-m f(M)}{M-m},
$$

so the linear function through $(m, f(m))$ and $(M, f(M))$ has the form $h(z)=$ $k_{f} z+l_{f}$.

2.1. Auxiliary results. To prove our main results we need the following lemmas.

Lemma 2.1. (a special case of [7, Lemma 4]) Let $A$ be a self-adjoint operator on a Hilbert space $H$ such that $m 1_{H} \leq A \leq M 1_{H}$ for some scalars $m<M$. If $f$ is a convex function on $[m, M]$, then

$$
f(A) \leq k_{f} A+l_{f}-\delta_{f} \tilde{A} \leq k_{f} A+l_{f},
$$


where

$$
\tilde{A}:=\frac{1}{2} 1_{H}-\frac{1}{M-m}\left|A-\frac{m+M}{2} 1_{H}\right| .
$$

If $f$ is concave, then the reverse inequality is valid in (2.1).

Proof. For the sake of completeness, we give a direct proof here. We prove the convex case only. We use known result (see [7, Lemma 3]): If $f$ is a convex function on an interval $I, m, M \in I$ and $p_{1}, p_{2} \in[0,1]$ such that $p_{1}+p_{2}=1$, then

$$
\begin{aligned}
& f\left(p_{1} m+p_{2} M\right) \\
\leq & p_{1} f(m)+p_{2} f(M)-\min \left\{p_{1}, p_{2}\right\}\left[f(m)+f(M)-2 f\left(\frac{m+M}{2}\right)\right] .
\end{aligned}
$$

Let functions $p_{1}, p_{2}:[m, M] \rightarrow[0,1]$ be defined by $p_{1}(z)=\frac{M-z}{M-m}, p_{2}(z)=\frac{z-m}{M-m}$. Then for any $z \in[m, M]$ we can write $f(z)=f\left(p_{1}(z) m+p_{2}(z) M\right)$. Using (2.2) and since $\bar{z}:=\min \left\{\frac{M-z}{M-m}, \frac{z-m}{M-m}\right\}=\frac{1}{2}-\frac{1}{M-m}\left|z-\frac{m+M}{2}\right|$, we get

$$
f(z) \leq \frac{M-z}{M-m} f(m)+\frac{z-m}{M-m} f(M)-\bar{z}\left[f(m)+f(M)-2 f\left(\frac{m+M}{2}\right)\right] .
$$

Now since $\operatorname{Sp}(A) \subseteq[m, M]$, then by utilizing the functional calculus to (2.3) we obtain LHS of (2.1).

Since $m 1_{H} \leq A \leq M 1_{H}$ then $-\frac{M-m}{2} 1_{H} \leq A-\frac{m+M}{2} 1_{H} \leq \frac{M-m}{2} 1_{H}$. It follows $\left|A-\frac{m+M}{2} 1_{H}\right| \leq \frac{M-m}{2} 1_{H}$, so $\tilde{A} \geq 0$. Also, since $f$ is convex then $\delta_{f} \geq 0$. Therefore, $\delta_{f} \tilde{A} \geq 0$ and RHS of (2.1) holds.

In the following lemma a refinement of inequalities [1, Theorem 2.9 and Theorem 2.21] is given.

Lemma 2.2. Let $A$ be a self-adjoint operator on a Hilbert space $H$ such that $m 1_{H} \leq A \leq M 1_{H}$ for some scalars $m<M$. Let $m_{\tilde{A}}$ be the lower bound of the operator $\tilde{A}=\frac{1}{2} 1_{H}-\frac{1}{M-m}\left|A-\frac{M+m}{2} 1_{H}\right|$. If $f$ is a convex function on $[m, M]$, then

$$
0 \leq\langle f(A) y, y\rangle-f(\langle A y, y\rangle) \leq C\left(m, M, f, \delta_{f} m_{\tilde{A}}\right) 1_{H} \leq C(m, M, f, 0) 1_{H},
$$

for every unit vector $y \in H$, where

$$
C(m, M, f, c)=\max _{m \leq z \leq M}\left\{k_{f} z+l_{f}-c-f(z)\right\}, \quad c \in \mathbb{R} .
$$

Additionally, if $f>0$ on $[m, M]$, then

$$
\begin{aligned}
& f(\langle A y, y\rangle) \leq\langle f(A) y, y\rangle \\
\leq \quad & K\left(m, M, f, \delta_{f} m_{\tilde{A}}\right) f(\langle A y, y\rangle) \leq K(m, M, f, 0) f(\langle A y, y\rangle)
\end{aligned}
$$

for every unit vector $y \in H$, where

$$
K(m, M, f, c)=\max _{m \leq z \leq M}\left\{\frac{k_{f} z+l_{f}-c}{f(z)}\right\}, \quad c \in \mathbb{R} .
$$

If $f$ is concave, then the reverse inequalities are valid in (2.4) and (2.6) with min instead of $\max$ in (2.5) and (2.7), respectively. 
Proof. We prove the convex case only. The first inequality in (2.4) is obvious. By using (2.1) and since $0 \leq m_{\tilde{A}} 1_{H} \leq \tilde{A}$ we have

$$
\langle f(A) y, y\rangle \leq k_{f}\langle A y, y\rangle+l_{f}-\left\langle\delta_{f} \tilde{A} y, y\right\rangle \leq k_{f}\langle A y, y\rangle+l_{f}-\delta_{f} m_{\tilde{A}} .
$$

It follows

$$
\langle f(A) y, y\rangle-f(\langle A y, y\rangle) \leq \max _{m \leq z \leq M}\left\{k_{f} z+l_{f}-\delta_{f} m_{\tilde{A}}-f(z)\right\} 1_{H}
$$

and hence we have the second inequality in (2.4). Since $\delta_{f} m_{\tilde{A}} \geq 0$ we have the third inequality in (2.4).

If $f>0$ on $[m, M]$, then divide inequalities (2.8) by $f(\langle A y, y\rangle)$ we get

$$
\frac{\langle f(A) y, y\rangle}{f(\langle A y, y\rangle)} \leq \frac{k_{f}\langle A y, y\rangle+l_{f}-\delta_{f} m_{\tilde{A}}}{f(\langle A y, y\rangle)} \leq \max _{m \leq z \leq M}\left\{\frac{k_{f} z+l_{f}-\delta_{f} m_{\tilde{A}}}{f(z)}\right\} 1_{H}
$$

and we have the second inequality in (2.6). Since $\delta_{f} m_{\tilde{A}} \geq 0$ we obtain the third inequality in (2.6).

By using the improvement of the Mond-Pečarić method given in Lemma 2.1 we present an auxiliary result for quasi-arithmetic means.

Lemma 2.3. Let $\left(x_{t}\right)_{t \in T}, m, M$ and $\left(\phi_{t}\right)_{t \in T}$ be as in the definition of the quasiarithmetic mean $(1.1)$ and $\varphi, \psi \in \mathcal{C}([m, M])$ be strictly monotone functions. If $\psi \circ \varphi^{-1}$ is convex, then

$$
\psi\left(\mathfrak{M}_{\psi}\right) \leq k_{1} \varphi\left(\mathfrak{M}_{\varphi}\right)+l_{1} 1_{K}-\delta_{1} \widetilde{x}_{\varphi} \leq k_{1} \varphi\left(\mathfrak{M}_{\varphi}\right)+l_{1} 1_{K}
$$

where $k_{1}=k_{\psi \circ \varphi^{-1}}(\varphi(m), \varphi(M)), l_{1}=l_{\psi \circ \varphi^{-1}}(\varphi(m), \varphi(M)), \delta_{1}=\delta_{\psi \circ \varphi^{-1}}(\varphi(m)$, $\varphi(M))$, and

$$
\widetilde{x}_{\varphi}:=\frac{1}{2} 1_{K}-\frac{1}{|\varphi(M)-\varphi(m)|} \int_{T} \phi_{t}\left(\left|\varphi\left(x_{t}\right)-\frac{\varphi(m)+\varphi(M)}{2} 1_{H}\right|\right) d \mu(t) .
$$

If $\psi \circ \varphi^{-1}$ is concave, then the reverse inequality is valid in (2.9).

Proof. We prove the convex case only. Setting $f=\psi \circ \varphi^{-1}$ and $A=\varphi\left(x_{t}\right)$ in (2.1), and since $\operatorname{Sp}\left(\varphi\left(x_{t}\right)\right) \subseteq[\varphi(m), \varphi(M)]$ if $\varphi$ is increasing or $\operatorname{Sp}\left(\varphi\left(x_{t}\right)\right) \subseteq$ $[\varphi(M), \varphi(m)]$ if $\varphi$ is decreasing, we obtain

$\psi\left(x_{t}\right) \leq k_{1} \varphi\left(x_{t}\right)+l_{1} 1_{H}-\delta_{1}\left(\frac{1}{2} 1_{H}-\frac{1}{|\varphi(M)-\varphi(m)|}\left|\varphi\left(x_{t}\right)-\frac{\varphi(m)+\varphi(M)}{2} 1_{H}\right|\right)$.

Applying a positive linear mapping $\phi_{t}$, integrating and using $\int_{T} \phi_{t}\left(1_{H}\right) d \mu(t)=1_{K}$, we obtain LHS of (2.9). Since $\delta_{1} \widetilde{x}_{\varphi} \geq 0$, RHS of (2.9) holds.

2.2. Ratio type inequalities. In this subsection ratio type inequalities involving quasi-arithmetic means are given. In the following theorem is given a complementary result to (1.2) without operator convexity of $\psi \circ \varphi^{-1}$.

Theorem 2.4. Let $\left(x_{t}\right)_{t \in T}, m, M$ and $\left(\phi_{t}\right)_{t \in T}$ be as in the definition of the quasiarithmetic mean (1.1), $0<m<M$ and $\varphi, \psi \in \mathcal{C}([m, M])$ be strictly monotone functions. Let $k_{1}, l_{1}, \delta_{1}$ and $\widetilde{x}_{\varphi}$ be as in Lemma 2.3. If one of the following conditions 
(4-i) : $\psi \circ \varphi^{-1}$ is convex and $\psi^{-1}$ is operator monotone

(4-ii): $\psi \circ \varphi^{-1}$ is concave and $-\psi^{-1}$ is operator monotone

is satisfied, then

$$
\mathfrak{M}_{\psi} \leq K_{1}\left(m_{\varphi}, M_{\varphi}, m_{\widetilde{x}_{\varphi}}\right) \mathfrak{M}_{\varphi} \leq K_{1}\left(m_{\varphi}, M_{\varphi}, 0\right) \mathfrak{M}_{\varphi} \leq K_{1}(m, M, 0) \mathfrak{M}_{\varphi}
$$

where $m_{\widetilde{x}_{\varphi}}$ is the lower bound of the operator $\widetilde{x}_{\varphi}, m_{\varphi}$ and $M_{\varphi}$ are the lower and upper bounds of the mean $\mathfrak{M}_{\varphi}$, respectively, and

$$
K_{1}(n, N, c):=\max _{n \leq z \leq N}\left\{\frac{\psi^{-1}\left(k_{1} \varphi(z)+l_{1}-c \delta_{1}\right)}{z}\right\} .
$$

If one of the following conditions

(4-iii) : $\psi \circ \varphi^{-1}$ is concave and $\psi^{-1}$ is operator monotone

(4-iv): $\psi \circ \varphi^{-1}$ is convex and $-\psi^{-1}$ is operator monotone

is satisfied, then the reverse inequalities are valid in (2.11) with min instead of $\max$ in (2.12).

Proof. We prove only the case (4-i). Applying operator monotone function $\psi^{-1}$ to inequalities $(2.9)$ and since $0 \leq m_{\widetilde{x}_{\varphi}} 1_{K} \leq \widetilde{x}_{\varphi}$, we have

$$
\begin{aligned}
\mathfrak{M}_{\psi} & \leq \psi^{-1}\left(k_{1} \varphi\left(\mathfrak{M}_{\varphi}\right)+l_{1} 1_{K}-\delta_{1} \widetilde{x}_{\varphi}\right) \leq \psi^{-1}\left(k_{1} \varphi\left(\mathfrak{M}_{\varphi}\right)+l_{1} 1_{K}-\delta_{1} m_{\widetilde{x}_{\varphi}} 1_{K}\right) \\
& \leq \psi^{-1}\left(k_{1} \varphi\left(\mathfrak{M}_{\varphi}\right)+l_{1} 1_{K}\right) .
\end{aligned}
$$

It follows

$$
\begin{aligned}
\mathfrak{M}_{\psi} & \leq \max _{m_{\varphi} \leq z \leq M_{\varphi}}\left\{\frac{\psi^{-1}\left(k_{1} \varphi(z)+l_{1}-\delta_{1} m_{\tilde{x} \varphi}\right)}{z}\right\} \mathfrak{M}_{\varphi} \\
& \leq \max _{m_{\varphi} \leq z \leq M_{\varphi}}\left\{\frac{\psi^{-1}\left(k_{1} \varphi(z)+l_{1}\right)}{z}\right\} \mathfrak{M}_{\varphi} \leq \max _{m \leq z \leq M}\left\{\frac{\psi^{-1}\left(k_{1} \varphi(z)+l_{1}\right)}{z}\right\} \mathfrak{M}_{\varphi}
\end{aligned}
$$

since $m 1_{K} \leq m_{\varphi} 1_{K} \leq \mathfrak{M}_{\varphi} \leq M_{\varphi} 1_{K} \leq M 1_{K}$. Hence we have the desired inequalities (2.11).

In the following theorem a complementary result to (1.2) is presented without operator monotonicity of $\psi^{-1}$.

Theorem 2.5. Let $\left(x_{t}\right)_{t \in T}, m, M$ and $\left(\phi_{t}\right)_{t \in T}$ be as in the definition of the quasiarithmetic mean (1.1), $0<m<M$ and $\varphi, \psi \in \mathcal{C}([m, M])$ be strictly monotone functions. If one of the following conditions

(5-i) : $\psi \circ \varphi^{-1}$ is operator convex and $\psi^{-1}$ is increasing convex

(5-ii): $\psi \circ \varphi^{-1}$ is operator concave and $\psi^{-1}$ is decreasing convex

is satisfied, then

$$
\mathfrak{M}_{\varphi} \leq K_{2}\left(m_{\varphi}, M_{\varphi}, m_{\widetilde{x}_{\varphi}}\right) \mathfrak{M}_{\psi} \leq K_{2}\left(m_{\varphi}, M_{\varphi}, 0\right) \mathfrak{M}_{\psi} \leq K_{2}(m, M, 0) \mathfrak{M}_{\psi},
$$

where $m_{\varphi}$ and $M_{\varphi}$ are the lower and upper bounds of the mean $\mathfrak{M}_{\varphi}$, respectively, $m_{\widetilde{x}_{\varphi}}$ is the lower bound of the operator

$$
\widetilde{x}_{\varphi, \psi}=\frac{1}{2} 1_{K}-\frac{1}{\left|\psi\left(M_{\varphi}\right)-\psi\left(m_{\varphi}\right)\right|}\left|\psi\left(\mathfrak{M}_{\varphi}\right)-\frac{\psi\left(m_{\varphi}\right)+\psi\left(M_{\varphi}\right)}{2} 1_{K}\right|,
$$


$k_{2}=k_{\psi^{-1}}\left(\psi\left(m_{\varphi}\right), \psi\left(M_{\varphi}\right)\right), l_{2}=l_{\psi^{-1}}\left(\psi\left(m_{\varphi}\right), \psi\left(M_{\varphi}\right)\right), \delta_{2}=\delta_{\psi^{-1}}\left(\psi\left(m_{\varphi}\right), \psi\left(M_{\varphi}\right)\right)$, and

$$
K_{2}(n, N, c):=\max _{z \in I}\left\{\frac{k_{2} z+l_{2}-c \delta_{2}}{\psi^{-1}(z)}\right\},
$$

where $I$ is the closed interval between $\psi(n)$ and $\psi(N)$.

If one of the following conditions

(5-iii) : $\psi \circ \varphi^{-1}$ is operator convex and $\psi^{-1}$ is decreasing concave

(5-iv): $\psi \circ \varphi^{-1}$ is operator concave and $\psi^{-1}$ is increasing concave

is satisfied, then the reverse inequalities are valid in (2.13) with min instead of $\max$ in (2.14).

If one of the following conditions

(5-v) : $\psi \circ \varphi^{-1}$ is operator convex and $\psi^{-1}$ is decreasing convex

(5-vi): $\psi \circ \varphi^{-1}$ is operator concave and $\psi^{-1}$ is increasing convex is satisfied, then

$$
\mathfrak{M}_{\psi} \leq K_{3}\left(m_{\psi}, M_{\psi}, m_{\widetilde{x}_{\psi}}\right) \mathfrak{M}_{\varphi} \leq K_{3}\left(m_{\psi}, M_{\psi}, 0\right) \mathfrak{M}_{\varphi} \leq K_{3}(m, M, 0) \mathfrak{M}_{\varphi}
$$

where $m_{\psi}$ and $M_{\psi}$ are the lower and upper bounds of the mean $\mathfrak{M}_{\psi}$, respectively, $m_{\tilde{x}_{\psi}}$ is the lower bound of the operator

$$
\begin{aligned}
& \qquad \widetilde{x}_{\psi}=\frac{1}{2} 1_{K}-\frac{1}{\left|\psi\left(M_{\psi}\right)-\psi\left(m_{\psi}\right)\right|}\left|\psi\left(\mathfrak{M}_{\psi}\right)-\frac{\psi\left(m_{\psi}\right)+\psi\left(M_{\psi}\right)}{2} 1_{K}\right|, \\
& k_{3}=k_{\psi^{-1}}\left(\psi\left(m_{\psi}\right), \psi\left(M_{\psi}\right)\right), l_{3}=l_{\psi^{-1}}\left(\psi\left(m_{\psi}\right), \psi\left(M_{\psi}\right)\right), \delta_{3}=\delta_{\psi^{-1}}\left(\psi\left(m_{\psi}\right), \psi\left(M_{\psi}\right)\right), \\
& \text { and } \\
& \qquad K_{3}(n, N, c):=\max _{z \in I}\left\{\frac{k_{3} z+l_{3}-c \delta_{3}}{\psi^{-1}(z)}\right\}
\end{aligned}
$$

where $I$ is the closed interval between $\psi(n)$ and $\psi(N)$.

If one of the following conditions

(5-vii) : $\psi \circ \varphi^{-1}$ is operator convex and $\psi^{-1}$ is decreasing concave

(5-viii): $\psi \circ \varphi^{-1}$ is operator concave and $\psi^{-1}$ is increasing concave

is satisfied, then the reverse inequalities are valid in (2.15) with min instead of $\max$ in (2.16).

Proof. (5-i): Replacing $f$ by a convex function $\psi^{-1}$ and $A$ by $\psi\left(\mathfrak{M}_{\varphi}\right)$ in RHS of (2.6), we obtain

$$
\left\langle\mathfrak{M}_{\varphi} y, y\right\rangle=\left\langle\psi^{-1}\left(\psi\left(\mathfrak{M}_{\varphi}\right)\right) y, y\right\rangle \leq K_{2}\left(m_{\varphi}, M_{\varphi}, m_{\widetilde{x}_{\psi}}\right) \psi^{-1}\left(\left\langle\psi\left(\mathfrak{M}_{\varphi}\right) y, y\right\rangle\right)
$$

for every unit vector $y \in H$, where

$$
\begin{aligned}
K_{2}\left(m_{\varphi}, M_{\varphi}, m_{\widetilde{x}_{\varphi}}\right) & =K\left(\psi\left(m_{\varphi}\right), \psi\left(M_{\varphi}\right), \psi^{-1}, m_{\widetilde{x}_{\varphi}}\right) \\
& =\max _{\psi\left(m_{\varphi}\right) \leq z \leq \psi\left(M_{\varphi}\right)}\left\{\frac{k_{2} z+l_{2}-\delta_{2} m_{\tilde{x}_{\varphi}}}{\psi^{-1}(z)}\right\}>0 .
\end{aligned}
$$

By using Jensen's operator inequality for an operator convex function $\psi \circ \varphi^{-1}$ we have

$$
\psi\left(\mathfrak{M}_{\varphi}\right)=\psi \circ \varphi^{-1}\left(\int_{T} \phi_{t}\left(\varphi\left(x_{t}\right)\right) d \mu(t)\right) \leq \int_{T} \phi_{t}\left(\psi\left(x_{t}\right)\right) d \mu(t)=\psi\left(\mathfrak{M}_{\psi}\right)
$$


Replacing $f$ by $\psi^{-1}$ and $A$ by $\psi\left(\mathfrak{M}_{\psi}\right)$ in LHS of (2.6), we obtain

$$
\psi^{-1}\left(\left\langle\psi\left(\mathfrak{M}_{\psi}\right) y, y\right\rangle\right) \leq\left\langle\mathfrak{M}_{\psi} y, y\right\rangle
$$

for every unit vector $y \in H$. Finally, we obtain

$$
\begin{aligned}
\frac{1}{K_{2}\left(m_{\varphi}, M_{\varphi}, m_{\tilde{x}_{\psi}}\right)}\left\langle\mathfrak{M}_{\varphi} y, y\right\rangle & \leq \psi^{-1}\left(\left\langle\psi\left(\mathfrak{M}_{\varphi}\right) y, y\right\rangle\right) & & \text { by }(2.17) \\
& \leq \psi^{-1}\left(\left\langle\psi\left(\mathfrak{M}_{\psi}\right) y, y\right\rangle\right) & & \text { by }(2.18) \text { and increase of } \psi^{-1} \\
& \leq\left\langle\mathfrak{M}_{\psi} y, y\right\rangle & & \text { by }(2.19)
\end{aligned}
$$

for each unit vector $y \in H$ and hence we have the first inequality in (2.13). The remaining inequalities in (2.13) are obvious.

(5-ii)-(5-iv): We obtain these results by a similar method as in (5-i).

(5-v): Replacing $f$ by a convex function $\psi^{-1}$ and $A$ by $\psi\left(\mathfrak{M}_{\psi}\right)$ in RHS of (2.6), we obtain

$$
\left\langle\mathfrak{M}_{\psi} y, y\right\rangle=\left\langle\psi^{-1}\left(\psi\left(\mathfrak{M}_{\psi}\right)\right) y, y\right\rangle \leq K_{3}\left(m_{\psi}, M_{\psi}, m_{\widetilde{x}_{\psi}}\right) \psi^{-1}\left(\left\langle\psi\left(\mathfrak{M}_{\psi}\right) y, y\right\rangle\right)
$$

for every unit vector $y \in H$, where

$$
\begin{aligned}
K_{3}\left(m_{\psi}, M_{\psi}, m_{\widetilde{x}_{\psi}}\right) & =K\left(\psi\left(m_{\psi}\right), \psi\left(M_{\psi}\right), \psi^{-1}, m_{\widetilde{x}_{\psi}}\right) \\
& =\max _{\psi\left(m_{\varphi}\right) \leq z \leq \psi\left(M_{\psi}\right)}\left\{\frac{k_{3} z+l_{3}-\delta_{3} m_{\widetilde{x}_{\psi}}}{\psi^{-1}(z)}\right\}>0 .
\end{aligned}
$$

Replacing $f$ by $\psi^{-1}$ and $A$ by $\psi\left(\mathfrak{M}_{\varphi}\right)$ in LHS of (2.6), we obtain

$$
\psi^{-1}\left(\left\langle\psi\left(\mathfrak{M}_{\varphi}\right) y, y\right\rangle\right) \leq\left\langle\mathfrak{M}_{\varphi} y, y\right\rangle
$$

for every unit vector $y \in H$.

Finally, we obtain

$$
\begin{aligned}
\frac{1}{K_{3}\left(m_{\psi}, M_{\psi}, m_{\tilde{x}_{\psi}}\right)}\left\langle\mathfrak{M}_{\psi} y, y\right\rangle & \leq \psi^{-1}\left(\left\langle\psi\left(\mathfrak{M}_{\psi}\right) y, y\right\rangle\right) & & \text { by }(2.20) \\
& \leq \psi^{-1}\left(\left\langle\psi\left(\mathfrak{M}_{\varphi}\right) y, y\right\rangle\right) & & \text { by }(2.18) \text { and decrease of } \psi^{-1} \\
& \leq\left\langle\mathfrak{M}_{\varphi} y, y\right\rangle & & \text { by }(2.21)
\end{aligned}
$$

for each unit vector $y \in H$ and hence we have the first inequality in (2.15). The remaining inequalities in (2.15) are obvious.

(5-vi)-(5-viii): We obtain these results by a similar method as in (5-v).

In the following theorem we point out another complementary result to (1.2) without operator monotonicity of $\psi^{-1}$.

Theorem 2.6. Let $\left(x_{t}\right)_{t \in T}, m, M$ and $\left(\phi_{t}\right)_{t \in T}$ be as in the definition of the quasiarithmetic mean (1.1), $0<m<M$ and $\varphi, \psi \in \mathcal{C}([m, M])$ be strictly monotone functions.

(6-i) : If $\varphi^{-1}$ is convex and $\psi^{-1}$ is operator convex, then

$$
\mathfrak{M}_{\psi} \leq K_{4}\left(m_{\varphi}, M_{\varphi}, m_{\widetilde{x}_{\varphi}}\right) \mathfrak{M}_{\varphi} \leq K_{4}\left(m_{\varphi}, M_{\varphi}, 0\right) \mathfrak{M}_{\varphi} \leq K_{4}(m, M, 0) \mathfrak{M}_{\varphi}
$$

where $m_{\widetilde{x}_{\varphi}}$ is the lower bound of the operator $\widetilde{x}_{\varphi}$ defined by (2.10), $m_{\varphi}$ and $M_{\varphi}$ are the lower and upper bounds of the mean $\mathfrak{M}_{\varphi}$, respectively, $k_{4}=k_{\varphi^{-1}}(\varphi(m), \varphi(M))$, $l_{4}=l_{\varphi^{-1}}(\varphi(m), \varphi(M)), \delta_{4}=\delta_{\varphi^{-1}}(\varphi(m), \varphi(M))$, and

$$
K_{4}(n, N, c):=\max _{n \leq z \leq N}\left\{\frac{k_{4} \varphi(z)+l_{4}-c \delta_{4}}{z}\right\} .
$$


(6-ii) : If $\varphi^{-1}$ is concave and $\psi^{-1}$ is operator concave, then the reverse inequalities are valid in (2.22) with min instead of max in (2.23).

(6-iii) : If $\varphi^{-1}$ is convex and $\psi^{-1}$ is concave, then

$$
\mathfrak{M}_{\psi} \leq \frac{K_{4}\left(m_{\varphi}, M_{\varphi}, m_{\widetilde{x}_{\varphi}}\right)}{K_{5}\left(m_{\psi}, M_{\psi}, m_{\widetilde{x}_{\psi}}\right)} \mathfrak{M}_{\varphi}
$$

where $m_{\tilde{x}_{\psi}}, m_{\psi}, M_{\psi}, k_{5}, l_{5}, \delta_{5}$ are as constants in $(6-\mathrm{i})$ with replacing $\varphi$ by $\psi$, and

$$
K_{5}(n, N, c):=\min _{n \leq z \leq N}\left\{\frac{k_{5} \psi(z)+l_{5}-c \delta_{5}}{z}\right\} .
$$

(6-iv) : If $\varphi^{-1}$ is concave and $\psi^{-1}$ is operator convex, then

$$
\mathfrak{M}_{\psi} \leq \frac{1}{K_{6}\left(m_{\varphi}, M_{\varphi}, m_{\widetilde{x}_{\varphi}}\right)} \mathfrak{M}_{\varphi} \leq \frac{1}{K_{6}\left(m_{\varphi}, M_{\varphi}, 0\right)} \mathfrak{M}_{\varphi} \leq \frac{1}{K_{6}(m, M, 0)} \mathfrak{M}_{\varphi}
$$

where $m_{\varphi}$ and $M_{\varphi}$ are the lower and upper bounds of the operator $\varphi\left(\mathfrak{M}_{\varphi}\right)$, respectively, $m_{\widetilde{x}_{\varphi}}$ is the lower bound of the operator

$$
\widetilde{x}_{\varphi}=\frac{1}{2} 1_{K}-\frac{1}{M_{\varphi}-m_{\varphi}}\left|\varphi\left(\mathfrak{M}_{\varphi}\right)-\frac{m_{\varphi}+M_{\varphi}}{2} 1_{K}\right|,
$$

$k_{6}=k_{\varphi^{-1}}\left(\varphi\left(m_{\varphi}\right), \varphi\left(M_{\varphi}\right)\right), l_{6}=l_{\varphi^{-1}}\left(\varphi\left(m_{\varphi}\right), \varphi\left(M_{\varphi}\right)\right), \delta_{6}=\delta_{\varphi^{-1}}\left(\varphi\left(m_{\varphi}\right), \varphi\left(M_{\varphi}\right)\right)$, and

$$
K_{6}(n, N, c):=\min _{z \in I}\left\{\frac{k_{6} z+l_{6}-c \delta_{6}}{\varphi^{-1}(z)}\right\},
$$

where $I$ is the closed interval between $\varphi(n)$ and $\varphi(N)$.

(6-v) : If $\varphi^{-1}$ is convex and $\psi^{-1}$ is operator concave, then the reverse inequalities are valid in (2.25) with max instead of min in (2.26).

Proof. (6-i): Using (1.2), we obtain

$$
\mathfrak{M}_{\psi} \leq \mathfrak{M}_{\mathcal{I}}
$$

where $\mathcal{I}$ is the identity function. Replacing $\psi$ by $\mathcal{I}$ in Theorem 2.4 (i), then (2.11) gives

$$
\mathfrak{M}_{\mathcal{I}} \leq K_{3}\left(m_{\varphi}, M_{\varphi}, m_{\widetilde{x}_{\varphi}}\right) \mathfrak{M}_{\varphi} \leq K_{3}\left(m_{\varphi}, M_{\varphi}, 0\right) \mathfrak{M}_{\varphi} \leq K_{3}(m, M, 0) \mathfrak{M}_{\varphi}
$$

Combining (2.27) and (2.28), we have the desired inequality (2.22).

(6-ii): We obtain this result by a similar method as in (6-i).

(6-iii): We obtain (2.24) by combining (i) and (6-ii).

(6-iv): Replacing $f$ by $\varphi^{-1}>0$ and $A$ by $\varphi\left(\mathfrak{M}_{\varphi}\right)$ in

$\langle f(A) y, y\rangle \geq K\left(m, M, f, \delta_{f} m_{\tilde{A}}\right) f(\langle A y, y\rangle)$ (see $\left.(2.6)\right)$, we obtain

$$
\left\langle\mathfrak{M}_{\varphi} y, y\right\rangle=\left\langle\varphi^{-1}\left(\varphi\left(\mathfrak{M}_{\varphi}\right)\right) y, y\right\rangle \geq K_{6}\left(m_{\varphi}, M_{\varphi}, m_{\widetilde{x}_{\varphi}}\right) \varphi^{-1}\left(\left\langle\varphi\left(\mathfrak{M}_{\varphi}\right) y, y\right\rangle\right)
$$

for every unit vector $y \in H$.

Next, we consider the mapping $\Phi: C B(T, \mathcal{A}) \rightarrow M(\mathcal{B}) \subseteq B(K)$ from normed involutive algebra of of bounded continuous functions on $T$ with values in $\mathcal{A}$ to the multiplier algebra $M(\mathcal{B})$ acting on $K$ defined by setting $\Phi(X)=\int_{T} \phi_{t}\left(x_{t}\right) d \mu(t)$, where $X=\left(x_{t}\right)_{t \in T}$. Note that $\Phi$ is a unital positive linear map. Also $\varphi\left(\int_{T} x_{t} d \mu(t)\right)$ 
$=\int_{T} \varphi\left(x_{t}\right) d \mu(t)$ for every linear functional $\varphi$ in the norm dual $\mathcal{A}^{*}$, cf. [3]. Stinespring decomposition theorem ensures that $\Phi$ can be written in the form $\Phi(X)=V^{*} \pi(X) V$, where $\pi$ is a $*$-homomorphism to $B(H)$, and $V$ is an isometry on $H$. It follows that for each unit vector $y \in H$

$$
\begin{aligned}
& \varphi^{-1}\left(\left\langle\varphi\left(\mathfrak{M}_{\varphi}\right) y, y\right\rangle\right)=\varphi^{-1}(\langle\Phi(\varphi(X)) y, y\rangle)=\varphi^{-1}(\langle\pi(\varphi(X)) V y, V y\rangle) \\
\geq & \left\langle\varphi^{-1}(\pi(\varphi(X))) V y, V y\right\rangle \quad \text { by }\|V y\|=1 \text { and concavity of } \varphi^{-1} \\
= & \langle\pi(X) V y, V y\rangle=\langle\Phi(X) y, y\rangle=\left\langle\mathfrak{M}_{\mathcal{I}} y, y\right\rangle .
\end{aligned}
$$

So,

$$
\varphi^{-1}\left(\left\langle\varphi\left(\mathfrak{M}_{\varphi}\right) y, y\right\rangle\right) \geq\left\langle\mathfrak{M}_{\mathcal{I}} y, y\right\rangle .
$$

Combining (2.29) and (2.30), we have

$$
\mathfrak{M}_{\varphi} \geq K_{6}\left(m_{\varphi}, M_{\varphi}, m_{\widetilde{x}_{\varphi}}\right) \mathfrak{M}_{\mathcal{I}}
$$

Now combining (2.31) and (2.27), we have the the first inequality in (2.25). The remaining inequalities in (2.25) are obvious.

(6-v): We obtain this result by a similar method as in (6-iv).

2.3. Difference type inequalities. Similar to the above, in this section difference type inequalities involving quasi-arithmetic means are given. The proofs are similar to the above proofs and we omit details.

Theorem 2.7. Let all assumptions of Theorem 2.4 hold, except that $m$ can be negative or 0 .

If (4-i) or (4-ii) is satisfied, then

$\mathfrak{M}_{\psi} \leq \mathfrak{M}_{\varphi}+C_{1}\left(m_{\varphi}, M_{\varphi}, m_{\widetilde{x}_{\varphi}}\right) 1_{K} \leq \mathfrak{M}_{\varphi}+C_{1}\left(m_{\varphi}, M_{\varphi}, 0\right) 1_{K} \leq \mathfrak{M}_{\varphi}+C_{1}(m, M, 0) 1_{K}$,

where

$$
C_{1}(n, N, c):=\max _{n \leq z \leq N}\left\{\psi^{-1}\left(k_{1} \varphi(z)+l_{1}-c \delta_{1}\right)-z\right\} .
$$

If (4-iii) or (4-iv) is satisfied, then the reverse inequalities are valid in (2.32) with min instead of max in (2.33).

Theorem 2.8. Let all assumptions of Theorem 2.5 hold, except that $m$ can be negative or 0 .

If (5-i) or (5-ii) is satisfied, then

$\mathfrak{M}_{\varphi} \leq \mathfrak{M}_{\varphi}+C_{2}\left(m_{\varphi}, M_{\varphi}, m_{\widetilde{x}_{\varphi}}\right) 1_{K} \leq \mathfrak{M}_{\varphi}+C_{2}\left(m_{\varphi}, M_{\varphi}, 0\right) 1_{K} \leq \mathfrak{M}_{\varphi}+C_{2}(m, M, 0) 1_{K}$,

where

$$
C_{2}(n, N, c):=\max _{z \in I}\left\{k_{2} z+l_{2}-c \delta_{2}-\psi^{-1}(z)\right\}
$$

where $I$ is the closed interval between $\psi(n)$ and $\psi(N)$.

If (5-iii) or (5-iv) is satisfied, then the reverse inequalities are valid in (2.34) with min instead of $\max$ in (2.35). 
If (5-v) or (5-vi) is satisfied, then

$\mathfrak{M}_{\psi} \leq \mathfrak{M}_{\varphi}+C_{3}\left(m_{\psi}, M_{\psi}, m_{\widetilde{x}_{\psi}}\right) 1_{K} \leq \mathfrak{M}_{\varphi}+C_{3}\left(m_{\psi}, M_{\psi}, 0\right) 1_{K} \leq \mathfrak{M}_{\varphi}+C_{3}(m, M, 0) 1_{K}$,

where

$$
C_{3}(n, N, c):=\max _{z \in I}\left\{k_{3} z+l_{3}-c \delta_{3}-\psi^{-1}(z)\right\}
$$

where $I$ is the closed interval between $\psi(n)$ and $\psi(N)$.

If (5-vii) or (5-viii) is satisfied, then the reverse inequalities are valid in (2.36) with min instead of max in (2.37).

Theorem 2.9. Let all assumptions of Theorem 2.5 hold, except that $m$ can be negative or 0 .

If (6-i) is satisfied, then

$\mathfrak{M}_{\psi} \leq \mathfrak{M}_{\varphi}+C_{4}\left(m_{\varphi}, M_{\varphi}, m_{\tilde{x}_{\varphi}}\right) 1_{K} \leq \mathfrak{M}_{\varphi}+C_{4}\left(m_{\varphi}, M_{\varphi}, 0\right) 1_{K} \leq \mathfrak{M}_{\varphi}+C_{4}(m, M, 0) 1_{K}$,

where

$$
C_{4}(n, N, c):=\max _{n \leq z \leq N}\left\{k_{4} \varphi(z)+l_{4}-c \delta_{4}-z\right\} .
$$

If (6-ii) is satisfied, then the reverse inequalities are valid in (2.38) with min instead of max in (2.39).

If (6-iii) is satisfied, then

$$
\mathfrak{M}_{\psi} \leq \mathfrak{M}_{\varphi}+C_{4}\left(m_{\varphi}, M_{\varphi}, m_{\widetilde{x}_{\varphi}}\right) 1_{K}-C_{5}\left(m_{\psi}, M_{\psi}, m_{\widetilde{x}_{\psi}}\right) 1_{K}
$$

where

$$
C_{5}(n, N, c):=\min _{n \leq z \leq N}\left\{k_{5} \psi(z)+l_{5}-c \delta_{5}-z\right\} .
$$

If (6-iv) is satisfied, then

$\mathfrak{M}_{\psi} \leq \mathfrak{M}_{\varphi}-C_{6}\left(m_{\varphi}, M_{\varphi}, m_{\tilde{x}_{\varphi}}\right) 1_{K} \leq \mathfrak{M}_{\varphi}-C_{6}\left(m_{\varphi}, M_{\varphi}, 0\right) 1_{K} \leq \mathfrak{M}_{\varphi}-C_{6}(m, M, 0) 1_{K}$,

where

$$
C_{6}(n, N, c):=\min _{z \in I}\left\{k_{6} z+l_{6}-c \delta_{6}-\varphi^{-1}(z)\right\},
$$

where $I$ is the closed interval between $\varphi(n)$ and $\varphi(N)$.

If $(6-\mathrm{v})$ is satisfied, then the reverse inequalities are valid in (2.40) with max instead of min in (2.41).

\section{POWER MEANS}

In this section we refine selected inequalities involving power means defined as follows

$$
\mathfrak{M}_{r}(\mathbf{x}, \boldsymbol{\phi}):= \begin{cases}\left(\int_{T} \phi_{t}\left(x_{t}^{r}\right) \mathrm{d} \mu(t)\right)^{1 / r}, & r \in \mathbb{R} \backslash\{0\}, \\ \exp \left(\int_{T} \phi_{t}\left(\log x_{t}\right) \mathrm{d} \mu(t)\right), & r=0,\end{cases}
$$

where $\left(x_{t}\right)_{t \in T}$ is a bounded continuous field of strictly positive elements in a unital $C^{*}$-algebra $\mathcal{A}$ with the spectra in $[m, M], 0<m<M$, defined on a locally compact Hausdorff space $T$ equipped with a bounded Radon measure $\mu$ 
and $\left(\phi_{t}\right)_{t \in T}$ is a unital field of positive linear mappings $\phi_{t}: \mathcal{A} \rightarrow \mathcal{B}$ between $C^{*}$-algebras. The mean $\mathfrak{M}_{r}(\mathbf{x}, \phi)$ is briefly denoted by $\mathfrak{M}_{r}$.

In the following theorem we obtain a refinement of some inequalities given in [2, Theorem 4.4]. Also, in this case we give the explicit formula for the constant $K_{1}$ given in Theorem 2.4 .

Theorem 3.1. Let $\left(x_{t}\right)_{t \in T}, m, M$ and $\left(\phi_{t}\right)_{t \in T}$ be as in the definition of the power mean (3.1). Let $m_{r}$ and $M_{r}, m_{r} \leq M_{r}$, be the bounds of $\mathfrak{M}_{r}$, respectively, and $m_{\widetilde{x}_{r}}$ be the lower bound of the operator $\widetilde{x}_{r}=\frac{1}{2} 1_{K}-\frac{1}{\left|M^{r}-m^{r}\right|} \int_{T} \phi_{t}\left(\left|x_{t}^{r}-\frac{m^{r}+M^{r}}{2} 1_{H}\right|\right) d \mu(t)$.

If $0 \neq r \leq s, s \geq 1$ or $r \leq s \leq-1$, then

$$
\mathfrak{M}_{s} \leq \bar{\triangle}\left(m_{r}, M_{r}, r, s, m_{\widetilde{x}_{r}}\right) \mathfrak{M}_{r} \leq \bar{\triangle}\left(m_{r}, M_{r}, r, s, 0\right) \mathfrak{M}_{r} \leq \triangle\left(\frac{M}{m}, r, s, 0\right) \mathfrak{M}_{r},
$$

where

$$
\bar{\triangle}\left(m_{r}, M_{r}, r, s, c\right)=\left\{\begin{array}{lll}
\frac{\left(\bar{k}_{1} m_{r}^{r}+\bar{l}_{1}-c \bar{\delta}_{1}\right)^{1 / s}}{m_{r}} & \text { if } \quad \frac{s}{r} \frac{\bar{l}_{1}-c \bar{\delta}_{1}}{m_{r}} \geq\left(1-\frac{s}{r}\right) \bar{k}_{1}, \\
\triangle\left(\frac{M}{m}, r, s, c\right) & \text { if } \quad \frac{s}{r} \frac{\bar{l}_{1}-c \bar{\delta}_{1}}{m_{r}}<\left(1-\frac{s}{r}\right) \bar{k}_{1}<\frac{s}{r} \frac{\bar{l}_{1}-c \bar{\delta}_{1}}{M_{r}}, \\
\frac{\left(\bar{k}_{1} M_{r}^{r}+\bar{l}_{1}-c \bar{\delta}_{1}\right)^{1 / s}}{M_{r}} & \text { if } & \frac{s}{r} \frac{\bar{l}_{1}-c \bar{\delta}_{1}}{M_{r}} \leq\left(1-\frac{s}{r}\right) \bar{k}_{1},
\end{array}\right.
$$

with the abbreviations: $\bar{k}_{1}=\frac{M^{s}-m^{s}}{M^{r}-m^{r}}, \bar{l}_{1}=\frac{M^{r} m^{s}-m^{r} M^{s}}{M^{r}-m^{r}}, \bar{\delta}_{1}:=m^{s}+M^{s}-2^{1-\frac{s}{r}}\left(m^{r}+\right.$ $\left.M^{r}\right)^{\frac{s}{r}}$ and $\triangle(h, r, s, c)$ is a generalized Specht ratio $\triangle(h, r, s)$ defined by

$$
\begin{aligned}
\triangle(h, r, s, c) & :=\left\{\frac{r}{s-r} \frac{h^{s}-h^{r}+c\left(h^{r}-1\right)\left[1+h^{s}-2^{1-s / r}\left(1+h^{r}\right)^{s / r}\right]}{h^{r}-1}\right\}^{1 / s} \\
& \times\left\{\frac{s}{r-s} \frac{h^{r}-h^{s}-c\left(h^{r}-1\right)\left[1+h^{s}-2^{1-s / r}\left(1+h^{r}\right)^{s / r}\right]}{h^{s}-1}\right\}^{-1 / r} .
\end{aligned}
$$

Proof. Setting $\varphi(t)=t^{r}$ and $\psi(t)=t^{s}$ in Theorem 2.4, we obtain

$$
0 \leq \mathfrak{M}_{s} \leq K_{1}\left(m_{r}, M_{r}, m_{\widetilde{x}_{r}}\right) \mathfrak{M}_{r} \leq K_{1}\left(m_{r}, M_{r}, 0\right) \mathfrak{M}_{r} \leq K_{1}(m, M, 0) \mathfrak{M}_{r}
$$

where

$$
K_{1}(n, N, c)=\max _{n \leq z \leq N}\left\{\frac{\left(\bar{k}_{1} z^{r}+\bar{l}_{1}-c \bar{\delta}_{1}\right)^{1 / s}}{z}\right\}=\max _{n \leq z \leq N}\left\{\left(\frac{\bar{k}_{1} z^{r}+\bar{l}_{1}-c \bar{\delta}_{1}}{z^{s}}\right)^{1 / s}\right\} .
$$

We have (according to [7, Corollary 13])

$$
\max _{n \leq z \leq N}\left\{\frac{\bar{k}_{1} z^{r}+\bar{l}_{1}-c \bar{\delta}_{1}}{z^{s}}\right\}=\bar{K}\left(m_{r}, M_{r}, m^{r}, M^{r}, \frac{s}{r}, c\right) \quad \text { for } s>1,
$$

where

$$
\bar{K}\left(m_{r}, M_{r}, m^{r}, M^{r}, \frac{s}{r}, c\right):= \begin{cases}\frac{\bar{k}_{1} m_{r}^{r}+\bar{l}_{1}-c \bar{\delta}_{1}}{m_{r}} & \text { if } \quad \frac{s}{r} \frac{\left.\bar{l}_{1}-c \bar{\delta}_{1}\right)}{m_{r}} \geq\left(1-\frac{s}{r}\right) \bar{k}_{1}, \\ K\left(m^{r}, M^{r}, \frac{s}{r}, c\right) & \text { if } \quad \frac{s}{r} \frac{\bar{l}_{1}-c \bar{\delta}_{1}}{m_{r}}<\left(1-\frac{s}{r}\right) \bar{k}_{1}<\frac{s}{r} \frac{\bar{l}_{1}-c \bar{\delta}_{1}}{M_{r}} \\ \frac{\bar{k}_{1} M_{r}^{r}+\bar{l}_{1}-c \bar{\delta}_{1}}{M_{r}} & \text { if } \quad \frac{s}{r} \frac{\bar{l}_{1}-c \bar{\delta}_{1}}{M_{r}} \leq\left(1-\frac{s}{r}\right) \bar{k}_{1}\end{cases}
$$


and $K(a, b, p, c)$ is a generalized Kantorovich constant

$$
\begin{aligned}
K(a, b, p, c) & :=\frac{\left.a b^{p}-b a^{p}+c\left(a^{p}+b^{p}-2^{1-p}(a+b)^{p}\right)\right)(b-a)}{(p-1)(b-a)} \\
& \times\left(\frac{p-1}{p} \frac{b^{p}-a^{p}}{\left.a b^{p}-b a^{p}+c\left(a^{p}+b^{p}-2^{1-p}(a+b)^{p}\right)\right)(b-a)}\right)^{p},
\end{aligned}
$$

for $a \neq b, p \in \mathbb{R}$ and $0 \leq c \leq 0.5$. Similarly, we have

$$
\min _{n \leq z \leq N}\left\{\frac{\bar{k}_{1} z^{r}+\bar{l}_{1}-c \bar{\delta}_{1}}{z^{s}}\right\}=\bar{k}\left(m_{r}, M_{r}, m^{r}, M^{r}, \frac{s}{r}, c\right) \quad \text { if } s<-1,
$$

with $\bar{k}\left(m_{r}, M_{r}, m^{r}, M^{r}, \frac{s}{r}, m_{\widetilde{x}_{r}}\right)$ which equals RHS in (3.6) with reverse inequality signs.

Using (3.4), (3.5), (3.8) and monotonicity of a function $\psi^{-1}(t)=t^{\frac{1}{s}}$ we obtain

$$
K_{1}(n, N, c)=\left(\max _{n \leq z \leq N}\left\{\frac{\bar{k}_{1} z^{r}+\bar{l}_{1}-c \bar{\delta}_{1}}{z^{s}}\right\}\right)^{1 / s}=\bar{K}\left(m_{r}, M_{r}, m^{r}, M^{r}, \frac{s}{r}, c\right)^{1 / s},
$$

which gives our constant (3.2), because we can check directly that

$$
K\left(m^{r}, M^{r}, \frac{s}{r}, c\right)^{1 / s}=\triangle\left(\frac{M}{m}, r, s, c\right) .
$$

Using that $\bar{\triangle}\left(m_{r}, M_{r}, r, s, c\right) \leq \triangle\left(\frac{M}{m}, r, s, c\right)$ and letting $r \rightarrow 0$ in Theorem 3.1 we obtain the following result.

Corollary 3.2. Let $m_{r}, M_{r}, m, M$ be as in Theorem 3.1 and $m_{\widetilde{x}_{0}}$ be the lower bound of the operator $\widetilde{x}_{0}=\frac{1}{2} 1_{K}-\frac{1}{|\log M-\log m|} \int_{T} \phi_{t}\left(\left|\log \left(x_{t}\right)-\frac{\log M+\log m}{2} 1_{H}\right|\right) d \mu(t)$.

If $s \geq 1$, then

$$
\mathfrak{M}_{s} \leq S\left(\frac{M_{r}}{m_{x_{0}}}, s, m_{\widetilde{x}_{0}}\right)^{1 / s} \mathfrak{M}_{0} \leq S\left(\frac{M_{r}}{m_{r}}, s, 0\right)^{1 / s} \mathfrak{M}_{0} \leq S\left(\frac{M}{m}, s, 0\right)^{1 / s} \mathfrak{M}_{0}
$$

where

$$
S(h, s, c)=\frac{h^{\frac{s}{h^{s}-1}}\left(1-c\left(h^{s / 2}-1\right)^{2}\right)}{\mathrm{e} \log h^{\frac{s}{h^{s}-1}}}
$$

is a generalized Specht ratio $S(h, s):=\frac{h^{\frac{s}{h^{s}-1}}}{\operatorname{elog} h^{h^{s}-1}}($ see $[2, \S 2.6])$.

To prove Corollary 3.2 it is sufficient to prove the following lemma.

Lemma 3.3. Let $h>0$. Then

$$
\lim _{r \rightarrow 0} \triangle(h, r, s, c)=S(h, s, c)^{1 / s},
$$

where $\triangle(h, r, s, c)$ and $S(h, s, c)$ are defined by (3.3) and (3.10), respectively. 
Proof. In the proof we use (3.9): $\triangle(h, r, s, c)=K\left(h^{r}, \frac{s}{r}, c\right)^{1 / s}$. We have

$$
\begin{aligned}
& \lim _{r \rightarrow 0} K\left(h^{r}, \frac{s}{r}, c\right)=\lim _{r \rightarrow 0} \frac{r}{s-r} \cdot \frac{h^{s}-h^{r}+c\left(h^{r}-1\right)\left(h^{s}+1-2^{1-\frac{s}{r}}\left(1+h^{r}\right)^{s / r}\right)}{h^{r}-1} \\
\times & \lim _{r \rightarrow 0}\left(\frac{s}{r-s} \cdot \frac{h^{r}-h^{s}+c\left(1-h^{r}\right)\left(h^{s}+1-2^{1-\frac{s}{r}}\left(1+h^{r}\right)^{s / r}\right)}{h^{s}-1}\right)^{-s / r} .
\end{aligned}
$$

(i):

$$
\begin{aligned}
& \lim _{r \rightarrow 0} \frac{r}{s-r} \cdot \frac{h^{s}-h^{r}+c\left(h^{r}-1\right)\left(h^{s}+1-2^{1-\frac{s}{r}}\left(1+h^{r}\right)^{s / r}\right)}{h^{r}-1} \\
= & \lim _{r \rightarrow 0} \frac{r}{h^{r}-1} \cdot\left(\lim _{r \rightarrow 0} \frac{h^{s}-h^{r}}{s-r}-\lim _{r \rightarrow 0} \frac{c\left(h^{r}-1\right)}{s-r}\left(h^{s}+1-2^{1-\frac{s}{r}}\left(1+h^{r}\right)^{s / r}\right)\right) \\
= & \frac{1}{\log h} \cdot\left(\frac{h^{s}-1}{s}+0 \cdot\left(h^{s}+1-2 h^{s / 2}\right)\right)=\frac{h^{s}-1}{s \log h}
\end{aligned}
$$

(ii):

$$
\begin{aligned}
& \lim _{r \rightarrow 0}\left(-\frac{s}{r}\right) \log \left(\frac{s}{r-s} \cdot \frac{h^{r}-h^{s}+c\left(1-h^{r}\right)\left(h^{s}+1-2^{1-\frac{s}{r}}\left(1+h^{r}\right)^{s / r}\right)}{h^{s}-1}\right) \\
= & -\frac{h^{s}-1+\left(-1+c\left(h^{s}+1-2 h^{s / 2}\right)\right) s \log h}{h^{s}-1} \\
= & -1+\frac{s \log h}{h^{s}-1}-\frac{c\left(h^{s}+1-2 h^{s / 2}\right) s \log h}{h^{s}-1} \\
\Longrightarrow & \lim _{r \rightarrow 0}\left(\frac{s}{r-s} \cdot \frac{h^{r}-h^{s}+c\left(1-h^{r}\right)\left(h^{s}+1-2^{1-\frac{s}{r}}\left(1+h^{r}\right)^{s / r}\right)}{h^{s}-1}\right)^{-s / r} \\
= & \frac{1}{\mathrm{e}} \frac{h^{\frac{s}{h^{s}-1}}}{h^{\frac{s c\left(h^{s / 2}-1\right)^{2}}{h^{s}-1}}}
\end{aligned}
$$

Combining (i) and (ii), we have

$$
\lim _{r \rightarrow 0} K\left(h^{r}, \frac{s}{r}, c\right)=\frac{h^{\frac{p}{h^{p}-1}\left(1-c\left(h^{s / 2}-1\right)^{2}\right)}}{\mathrm{e} \log h^{\frac{p}{h^{p}-1}}}=S(h, s, c) .
$$

Then, by using (3.9), we obtain

$$
\lim _{r \rightarrow 0} \triangle(h, r, s, c)=\lim _{r \rightarrow 0} K\left(h^{r}, \frac{s}{r}, c\right)^{1 / s}=S(h, s, c)^{1 / s} .
$$

In the following corollary we give the explicit formula for the constant $K_{2}$ given in Theorem 2.5.

Corollary 3.4. Let $\left(x_{t}\right)_{t \in T}, m, M$ and $\left(\phi_{t}\right)_{t \in T}$ be as in the definition of the power mean (3.1). Let $m_{r}$ and $M_{r}$ be the lower and upper bounds of the mean $\mathfrak{M}_{r}$, respectively, and $m_{\widetilde{x}_{s}}$ be the lower bound of the operator

$$
\widetilde{x}_{s}=\frac{1}{2} 1_{K}-\frac{1}{\left|M_{r}^{s}-m_{r}^{s}\right|}\left|\mathfrak{M}_{r}^{s}-\frac{m_{r}^{s}+M_{r}^{s}}{2} 1_{K}\right| .
$$




$$
\begin{aligned}
& \text { If } 0<s \leq-r \text { or } 0<r \leq s \leq 2 r \text { or } r \leq s<0 \text {, then } \\
& \qquad \begin{aligned}
\mathfrak{M}_{s} & \leq \bar{K}_{2}\left(m_{r}, M_{r}, m, M, m_{\widetilde{x}_{s}}\right) \mathfrak{M}_{r} \\
& \leq \bar{K}_{2}\left(m_{r}, M_{r}, m, M, 0\right) \mathfrak{M}_{r} \leq K(m, M, 1 / s, 0) \mathfrak{M}_{r}
\end{aligned}
\end{aligned}
$$

where

$$
\bar{K}_{2}\left(m_{r}, M_{r}, m, M, c\right)= \begin{cases}\frac{\bar{k}_{2} m_{r}+\bar{l}_{2}-c \bar{\delta}_{2}}{m_{r}^{1 / s}} & \text { if } \frac{\bar{l}_{2}-c \bar{\delta}_{2}}{s m_{r}} \geq\left(1-\frac{1}{s}\right) \bar{k}_{2}, \\ K(m, M, 1 / s, c) & \text { if } \frac{\bar{l}_{2}-c \bar{\delta}_{2}}{s m_{r}}<\left(1-\frac{1}{s}\right) \bar{k}_{2}<\frac{\bar{l}_{2}-c \bar{\delta}_{2}}{s M_{r}}, \\ \frac{\bar{k}_{2} M_{r}+\bar{l}_{2}-c \bar{\delta}_{2}}{M_{r}^{1 / s}} & \text { if } \frac{\bar{l}_{2}-c \bar{\delta}_{2}}{s M_{r}} \leq\left(1-\frac{1}{s}\right) \bar{k}_{2},\end{cases}
$$

with the abbreviations: $\quad \bar{k}_{2}=\frac{M_{r}-m_{r}}{M_{r}^{s}-m_{r}^{s}}, \quad \bar{l}_{2}=\frac{M_{r}^{s} m_{r}-m_{r}^{s} M_{r}}{M_{r}^{s}-m_{r}^{s}}, \quad \bar{\delta}_{2}=m_{r}+M_{r}-$ $2\left(\frac{m_{r}^{s}+M_{r}^{s}}{2}\right)^{1 / s}$ and $K(m, M, 1 / s, c)$ is the generalized Kantorovich constant defined by $(3.7)$.

Proof. We obtain this corollary by setting $\varphi(t)=t^{r}$ and $\psi(t)=t^{s}$ in Theorem 2.5. We omit the details of the construction constants.

Remark 3.5. Similar to the ratio case we can obtain difference type inequalities involving power means by using Theorem 2.7-Theorem 2.9.

For example, if $0 \neq r \leq s, s \geq 1$ or $r \leq s \leq-1$, then

$$
\begin{aligned}
\mathfrak{M}_{s} & \leq \mathfrak{M}_{r}+\bar{C}_{1}\left(m_{r}, M_{r}, r, s, m_{\widetilde{x}_{r}}\right) 1_{K} \\
& \leq \mathfrak{M}_{r}+\bar{C}_{1}\left(m_{r}, M_{r}, r, s, 0\right) 1_{K} \leq \mathfrak{M}_{r}+\bar{C}_{1}(m, M, r, s, 0) 1_{K},
\end{aligned}
$$

where

$$
\bar{C}_{1}(n, N, r, s, c)=\max _{n \leq z \leq N}\left\{\left(\bar{k}_{1} z^{r}+\bar{l}_{1}-c \bar{\delta}_{1}\right)^{1 / s}-z\right\}
$$

and $m_{r}, M_{r}, \bar{k}_{1}, \bar{l}_{1}, \bar{\delta}_{1}$ and $m_{\widetilde{x}_{r}}$ are as defined in Theorem 3.1.

Finally, we present some examples which illustrate some of the above inequalities.

Example 3.6. Setting $r=1 / 3$ and $s=1$ in Theorem 3.1 we observe inequalities involving power means $\mathfrak{M}_{1 / 3}(\mathbf{x}, \phi)$ and $\mathfrak{M}_{1}(\mathbf{x}, \boldsymbol{\phi})$. There is no general relation between these means under the operator order. Really, if $T=\{1,2\}$, $\Phi_{k}\left(\left(a_{i j}\right)_{1 \leq i, j \leq 3}\right)=\frac{1}{2}\left(a_{i j}\right)_{1 \leq i, j \leq 2}, \mu(\{k\})=1, k=1,2$ and

$$
\begin{aligned}
& X_{1}=\left(\begin{array}{lll}
6 & 1 & 1 \\
1 & 1 & 0 \\
1 & 0 & 1
\end{array}\right)^{3}=\left(\begin{array}{ccc}
242 & 45 & 45 \\
45 & 9 & 8 \\
45 & 8 & 9
\end{array}\right), \\
& X_{2}=\left(\begin{array}{ccc}
10 & -1 & -1 \\
-1 & 2 & 1 \\
-1 & 1 & 3
\end{array}\right)^{3}=\left(\begin{array}{ccc}
1047 & -142 & -157 \\
-142 & 31 & 37 \\
-157 & 37 & 53
\end{array}\right),
\end{aligned}
$$


then

$$
\begin{gathered}
\mathfrak{M}_{1 / 3}=\left(\Phi_{1}\left(X_{1}^{1 / 3}\right)+\Phi_{2}\left(X_{2}^{1 / 3}\right)\right)^{3}=\left(\begin{array}{cc}
512 & 0 \\
0 & 3.375
\end{array}\right) \\
\mathfrak{M}_{1}=\Phi_{1}\left(X_{1}\right)+\Phi_{2}\left(X_{2}\right)=\left(\begin{array}{cc}
644.5 & -48.5 \\
-48.5 & 20
\end{array}\right)
\end{gathered}
$$

and

$$
\mathfrak{M}_{1}-\mathfrak{M}_{1 / 3}=\left(\begin{array}{cc}
132.5 & -48.5 \\
-48.5 & 16.625
\end{array}\right) \nexists 0 .
$$

Furthermore, $0.247 I_{2} \leq X_{1} \leq 258.753 I_{2}, 2.562 I_{2} \leq X_{2} \leq 1091.310 I_{2}$ and let $m=0.247, M=1091.310$ (rounded to three decimal places). Also, $\mathfrak{M}_{1 / 3}$ has the bounds $m_{1 / 3}=3.375$ and $M_{1 / 3}=512$. Further, $\tilde{X}_{1 / 3}=\left(\begin{array}{cc}0.195 & 0.050 \\ 0.050 & 0.078\end{array}\right)$, so $m_{\tilde{X}_{1 / 3}}=0.059$. Also, $\bar{\delta}_{1}=765.723$. Taking all these into account we obtain

$$
\begin{array}{ll}
\tilde{\Delta}\left(m_{1 / 3}, M_{1 / 3}, 1 / 3,1, m_{\tilde{X}_{1 / 3}}\right) & =15.836 \\
\tilde{\Delta}\left(m_{1 / 3}, M_{1 / 3}, 1 / 3,1,0\right) & =29.241 \\
\Delta\left(\frac{M}{m}, r, s, 0\right) & =42.729
\end{array}
$$

and hence we have ratio type inequalities:

$$
\mathfrak{M}_{1}<15.836 \mathfrak{M}_{1 / 3}<29.241 \mathfrak{M}_{1 / 3}<42.729 \mathfrak{M}_{1 / 3}
$$

Similarly, applying (3.11), we obtain

$$
\begin{array}{ll}
\bar{C}_{1}\left(m_{1 / 3}, M_{1 / 3}, 1 / 3,1, m_{\tilde{X}_{1 / 3}}\right) & =486.683 \\
\bar{C}_{1}\left(m_{1 / 3}, M_{1 / 3}, 1 / 3,1,0\right)=\bar{C}_{1}(m, M, 1 / 3,1,0) & =532.049 .
\end{array}
$$

and hence we have difference type inequalities:

$$
\mathfrak{M}_{1}<\mathfrak{M}_{1 / 3}+486.683 I_{2}<\mathfrak{M}_{1 / 3}+532.049 I_{2} .
$$

Example 3.7. Under the same assumptions as in Example 1, we obtain inequalities involving means $\mathfrak{M}_{1 / 3}$ and $\mathfrak{M}_{2}$ (constants are rounded to three decimal places):

$$
\begin{aligned}
& \mathfrak{M}_{1 / 3}<\mathfrak{M}_{2}<59.289 \mathfrak{M}_{1 / 3}<97.127 \mathfrak{M}_{1 / 3}<290.967 \mathfrak{M}_{1 / 3} \\
& \mathfrak{M}_{1 / 3}<\mathfrak{M}_{2}<\mathfrak{M}_{1 / 3}+564.026 I_{2}<\mathfrak{M}_{1 / 3}+609.902 I_{2}
\end{aligned}
$$

We remark that there is no general relation between $\mathfrak{M}_{1 / 3}$ and $\mathfrak{M}_{2}$ under the operator order.

E.g. if $X_{1}=\left(\begin{array}{ccc}100 & 1 & 1 \\ 1 & 1 & 0 \\ 1 & 0 & 2\end{array}\right)^{3}$ and $X_{2}=\left(\begin{array}{ccc}2 & -1 & -1 \\ -1 & 1 & 0 \\ -1 & 0 & 3\end{array}\right)^{3}$, then $\mathfrak{M}_{1 / 3} \nsubseteq \mathfrak{M}_{2}$. 


\section{REFERENCES}

1. M. Fujii, J. Mićić Hot, J. Pečarić and Y. Seo, Recent Developments of Mond-Pečarić Method in Operator Inequalities, Monographs in Inequalities 4, Element, Zagreb, 2012.

2. T. Furuta, J. Mićić Hot, J. Pečarić and Y. Seo, Mond-Pečarić Method in Operator Inequalities, Monographs in Inequalities 1, Element, Zagreb, 2005.

3. F. Hansen, J. Pečarić and I.Perić, Jensen's operator inequality and its converses, Math. Scand. 100 (2007), 61-73.

4. O. Hirzallah, F. Kittaneh and M. Krnić, N. Lovričević and J. Pečarić, Refinements and reverses of means inequalities for Hilbert space operators, Banach J. Math. Anal. 7 (2013), $15-29$

5. J. Mićić, Z. Pavić and J. Pečarić, Jensen's inequality for operators without operator convexity, Linear Algebra Appl. 434 (2011), 1228-1237.

6. J. Mićić, J. Pečarić and Y. Seo, Order among quasi-arithmetic means of positive operators, Math. Reports 14(64) (2012), 71-86.

7. J. Mićić, J. Perić and J. Pečarić, Refined converses of Jensen's inequality for operators, J. Inequal. Appl. 2013:353 (2013), 1-20.

Faculty of Mechanical EngineEring And Naval Architecture, University of Zagreb, Ivana LuČIĆA 5, 10000 ZaGreb, CroAtia

E-mail address: jmicic@fsb.hr 